\title{
Principales resultados de la estadística sobre mortalidad por accidentes en México, 1997*
}

$\mathrm{P}$ ara 1997 los accidentes ocuparon el cuarto lugar como causa de muerte con una tasa de 37.9 por 100000 habitantes y un peso relativo de $8.1 \%$ respecto al total de defunciones del país (440 437).

Por la importancia de este hecho, la Dirección General de Estadística e Informática de la Secretaría de Salud presenta en esta ocasión los principales resultados de la estadística sobre mortalidad por accidentes para 1997, en 10 tabulados de resumen. El primero, dedicado a la tendencia que se ha presentado desde 1980 a la fecha para ambos sexos, muestra un descenso persistente de la tasa de casi $50 \%$. Se puede observar que la mortalidad es mayor en el sexo masculino, pero que ha ido descendiendo a lo largo del periodo hasta alcanzar un índice de sobremortalidad de 336.3 hombres por cada 100 mujeres.

El cuadro II, que se refiere a la mortalidad por grupos quinquenales de edad según sexo, hace evidente el incremento de las tasas con la edad y el predominio del sexo masculino en los grupos de 20 a 39 años, cuyo índice supera las 600 defunciones por cada 100 muertes femeninas.

El cuadro III presenta las causas de mortalidad por accidentes de acuerdo con los grupos de la lista básica. Se puede observar que de las 35876 muertes, $43 \%$ corresponde a los accidentes de transporte (clave E47), la inmensa mayoría de ellos por tráfico de vehículos de motor (clave E471). Las caídas accidentales (E50), así como el ahogamiento y la sumersión (E521), constituyen también causas frecuentes de muerte. Destaca asimismo que en el grupo de edad productiva se concentra $66 \%$ de las muertes accidentales. El cuadro IV muestra la distribución de las defunciones por causas de acuerdo con el sexo, así como el índice de sobremortalidad masculina según las causas, el cual resulta sumamente elevado en los casos de accidentes por maquinaria y proyectil de arma de fuego.

Los seis cuadros restantes muestran información por entidad federativa. El cuadro V presenta el volumen y la tasa de mortalidad para los principales grupos de causas; el cuadro VI contiene además el peso relativo que tienen las defunciones por accidentes respecto a la mortalidad total de cada entidad, lo cual se refleja en el número de orden que ocupan los mismos dentro de las principales causas de defunción.

Los cuadros VII y VIII desglosan las defunciones por el sitio donde éstas ocurrieron y por el lugar donde sucedió el accidente que condujo a la muerte, debido a la importancia que ello tiene desde el punto de vista epidemiológico y porque de este modo se puede deter- minar la oportunidad en la atención a los lesionados. Podrá observarse que sólo 29\% de las defunciones ocurrió en una unidad médica, lo que indica el nivel de acceso a los servicios, mientras que el resto sucedió en el hogar o en otro sitio. Por otra parte, en $43 \%$ de las defunciones la lesión ocurrió en la vía pública pues, como se vio con anterioridad, muchas de ellas se deben a accidentes de tráfico.

Finalmente los cuadros IX y $\mathrm{X}$ muestran la distribución de las defunciones por el mes en que éstas ocurrieron y la condición de derechohabiencia, a fin de observar el comportamiento de estas variables, que son captadas mediante el certificado de defunción y que tienen relación con la exposición al riesgo de sufrir un accidente por algunas causas, así como con el acceso a los servicios.

Es importante destacar que en las causas de defunción por accidentes también puede haber un subregistro cuya magnitud es difícil de precisar, sobre todo por los problemas que se presentan en el llenado del certificado de defunción; las implicaciones legales que conllevan las muertes accidentales, en algunas ocasiones repercuten en la codificación y la selección de la causa básica.

\footnotetext{
* Sección preparada por la Dirección General de Estadística e Informática de la Secretaría de Salud, México.
} 


\section{EVOLUCIÓN DE LA MORTALIDAD POR ACCIDENTES} SEGÚN SEXo. MÉXICO, 1980-1997

\begin{tabular}{|c|c|c|c|c|c|c|c|c|}
\hline \multirow[t]{2}{*}{ Año } & \multicolumn{2}{|c|}{ Total } & \multicolumn{2}{|c|}{ Hombres } & \multicolumn{2}{|c|}{ Mujeres } & \multirow{2}{*}{$\frac{N E}{\text { No. }}$} & \multirow[t]{2}{*}{ ISM } \\
\hline & No. & Tasa* & No. & Tasa* & No. & Tasa* & & \\
\hline 1980 & 48098 & 71.6 & 37984 & 112.4 & 9801 & 29.4 & 313 & 387.6 \\
\hline 1981 & 54963 & 79.8 & 42822 & 123.6 & 11631 & 34.0 & 510 & 368.2 \\
\hline 1982 & 52839 & 74.9 & 41653 & 117.4 & 10707 & 30.5 & 479 & 389.0 \\
\hline 1983 & 45890 & 63.5 & 36034 & 99.2 & 9422 & 26.2 & 434 & 382.4 \\
\hline 1984 & 46242 & 62.6 & 35987 & 96.8 & 9478 & 25.8 & 777 & 379.7 \\
\hline 1985 & 45504 & 60.2 & 34190 & 90.0 & 10928 & 29.1 & 386 & 312.9 \\
\hline 1986 & 43814 & 56.7 & 33983 & 87.5 & 9465 & 24.6 & 366 & 359.0 \\
\hline 1987 & 42798 & 54.2 & 33551 & 84.6 & 9012 & 22.9 & 235 & 372.3 \\
\hline 1988 & 41626 & 51.7 & 32419 & 80.1 & 9009 & 22.5 & 198 & 359.9 \\
\hline 1989 & 40154 & 48.9 & 31090 & 75.3 & 8877 & 21.7 & 187 & 350.2 \\
\hline 1990 & 39400 & 47.2 & 30261 & 73.0 & 8979 & 21.4 & 160 & 337.0 \\
\hline 1991 & 39020 & 45.8 & 30100 & 71.2 & 8808 & 20.6 & 112 & 341.7 \\
\hline 1992 & 38246 & 44.1 & 30014 & 69.7 & 8162 & 18.7 & 70 & 367.7 \\
\hline 1993 & 37024 & 41.9 & 28765 & 65.6 & 8218 & 18.5 & 41 & 350.0 \\
\hline 1994 & 37234 & 41.4 & 28970 & 64.9 & 8248 & 18.2 & 16 & 351.2 \\
\hline 1995 & 35567 & 38.8 & 27396 & 60.3 & 8143 & 17.6 & 28 & 336.4 \\
\hline 1996 & 35073 & 37.6 & 27339 & 59.2 & 7723 & 16.4 & 11 & 354.0 \\
\hline 1997 & 35876 & 37.9 & 27634 & 58.9 & 8218 & 17.2 & 24 & 336.3 \\
\hline
\end{tabular}

*Tasa por 100000 habitantes

NE: no especificado

ISM: índice de sobremortalidad masculina. Defunciones de hombres entre mujeres por 100

Fuente: Instituto N acional de Estadística, Geografía e Informática y Dirección General de Estadística e Informática de la Secretaría de Salud 


\section{Cuadro II}

MoRTALIDAD POR ACCIDENTES SEgún SEXo

Y GRUPOS DE EDAD. MÉXICO, 1997

\begin{tabular}{|c|c|c|c|c|c|c|c|c|}
\hline \multirow{3}{*}{$\begin{array}{l}\text { Grupos } \\
\text { de edad }\end{array}$} & \multirow{2}{*}{\multicolumn{2}{|c|}{ Total }} & \multicolumn{6}{|c|}{$S e \times 0$} \\
\hline & & & \multicolumn{2}{|c|}{ Hombres } & \multicolumn{2}{|c|}{ Mujeres } & \multirow{2}{*}{$\frac{N E}{\text { Defunciones }}$} & \multirow[t]{2}{*}{ ISM } \\
\hline & Defunciones & Tasa* & Defunciones & Tasa* & Defunciones & Tasa* & & \\
\hline Total & 35876 & 37.9 & 27634 & 58.9 & 8218 & 17.2 & 24 & 336.3 \\
\hline$<1$ & 1108 & $41.1^{\ddagger}$ & 647 & $47.6^{\ddagger}$ & 459 & $34.3^{\ddagger}$ & 2 & 141.0 \\
\hline $1 \mathrm{a} 4$ & 1934 & 21.9 & 1145 & 25.5 & 789 & 18.2 & 0 & 145.1 \\
\hline 5 a 9 & 1310 & 11.9 & 838 & 15.0 & 472 & 8.7 & 0 & 177.5 \\
\hline 10 a 14 & 1348 & 12.5 & 972 & 17.8 & 372 & 7.0 & 4 & 261.3 \\
\hline 15 a 19 & 2973 & 28.6 & 2363 & 45.2 & 610 & 11.8 & 0 & 387.4 \\
\hline 20 a 24 & 3798 & 39.7 & 3291 & 69.1 & 505 & 10.5 & 2 & 651.7 \\
\hline 25 a 29 & 3236 & 38.2 & 2805 & 67.0 & 429 & 10.0 & 2 & 653.8 \\
\hline 30 a 34 & 2885 & 39.5 & 2501 & 69.4 & 382 & 10.3 & 2 & 654.7 \\
\hline 35 a 39 & 2609 & 43.1 & 2239 & 75.3 & 368 & 11.9 & 2 & 608.4 \\
\hline 40 a 44 & 2119 & 44.3 & 1803 & 77.4 & 316 & 12.9 & 0 & 570.6 \\
\hline 45 a 49 & 1837 & 49.0 & 1543 & 84.8 & 293 & 15.2 & 1 & 526.6 \\
\hline 50 a 54 & 1512 & 50.3 & 1245 & 85.7 & 267 & 17.2 & 0 & 466.3 \\
\hline 55 a 59 & 1363 & 55.1 & 1081 & 91.3 & 282 & 21.9 & 0 & 383.3 \\
\hline 60 a 64 & 1351 & 67.4 & 1071 & 112.8 & 280 & 26.5 & 0 & 382.5 \\
\hline 65 a 69 & 1258 & 81.4 & 926 & 128.9 & 332 & 40.2 & 0 & 278.9 \\
\hline 70 a 74 & 1180 & 106.7 & 824 & 165.0 & 356 & 58.7 & 0 & 231.5 \\
\hline 75 a 79 & 1059 & 147.1 & 705 & 226.8 & 354 & 86.5 & 0 & 199.2 \\
\hline 80 a 84 & 889 & 215.6 & 516 & 311.8 & 373 & 151.1 & 0 & 138.3 \\
\hline 85 y más & 1729 & 494.0 & 805 & 689.9 & 923 & 395.6 & 1 & 87.2 \\
\hline $\mathrm{NE}$ & 378 & & 314 & & 56 & & 8 & \\
\hline
\end{tabular}

*Tasa por 100000 habitantes por cada grupo de edad

¥Tasa de menores de 1 año por 100000 nacidos vivos registrados

$N E$ : no especificado

ISM: índice de sobremortalidad masculina. D efunciones de hombres entre mujeres por 100

Fuente: Instituto N acional de Estadística, G eografía e Informática y Dirección General de Estadística e Informática de la Secretaría de Salud 


\section{Cuadro III}

Defunciones por accidentes por grupos de edad según Causas de la lista básica. México, 1997

\begin{tabular}{|c|c|c|c|c|c|c|c|c|c|}
\hline \multirow{2}{*}{$\begin{array}{l}\text { Clave } \\
\text { CIE-9 }\end{array}$} & \multirow[t]{2}{*}{ Causas } & \multirow[b]{2}{*}{ Total } & \multicolumn{6}{|c|}{ Grupos de edad } & \multirow[b]{2}{*}{$\mathrm{NE}$} \\
\hline & & & $<1$ & $1-4$ & $5-14$ & $15-34$ & $35-64$ & $65 y+$ & \\
\hline E47-E53 & Total accidentes & 35876 & 1108 & 1934 & 2658 & 12892 & 10791 & 6115 & 378 \\
\hline E47 & Accidentes de transporte & 15330 & 70 & 591 & 1222 & 6492 & 5059 & 1754 & 142 \\
\hline E470 & Accidentes de ferrocarril & 264 & 0 & 7 & 8 & 113 & 91 & 28 & 17 \\
\hline E471 & Accidentes de tráfico de vehículos de motor & 14642 & 70 & 576 & 1173 & 6208 & 4822 & 1668 & 125 \\
\hline E472 & Accidentes de otros vehículos de carretera & 286 & 0 & 6 & 31 & 92 & 103 & 54 & 0 \\
\hline E473 & Accidentes de transporte acuático & 48 & 0 & 0 & 7 & 28 & 13 & 0 & 0 \\
\hline E474 & Accidentes del transporte aéreo y espacial & 60 & 0 & 0 & 1 & 36 & 23 & 0 & 0 \\
\hline E479 & Las demás & 30 & 0 & 2 & 2 & 15 & 7 & 4 & 0 \\
\hline \multirow[t]{3}{*}{$\overline{E 48}$} & Envenenamiento accidental & 1113 & 45 & 81 & 54 & 471 & 342 & 105 & 15 \\
\hline & Envenenamiento accidental por drogas, medicamentos & & & & & & & & \\
\hline & y productos biológicos & 315 & 27 & 18 & 10 & 138 & 77 & 38 & 7 \\
\hline E481 & Envenenamiento accidental por otras sustancias sólidas y líquidas & 540 & 8 & 43 & 22 & 232 & 193 & 36 & 6 \\
\hline E482 & Envenenamiento accidental por gases y vapores & 258 & 10 & 20 & 22 & 101 & 72 & 31 & 2 \\
\hline \multirow[t]{2}{*}{ E49 } & Contratiempos durante la atención médica, reacciones anormales & & & & & & & & \\
\hline & y complicaciones ulteriores & 165 & 3 & 2 & 1 & 17 & 61 & 81 & 0 \\
\hline $\mathrm{E} 50$ & Caídas accidentales & 4275 & 44 & 122 & 150 & 709 & 1133 & 2088 & 29 \\
\hline E51 & Accidentes causados por el fuego & 701 & 25 & 95 & 66 & 188 & 179 & 143 & 5 \\
\hline \multirow{6}{*}{$\begin{array}{l}\mathrm{E} 5 \\
\mathrm{E} 5 \\
\mathrm{E} 5 \\
\mathrm{E} 5\end{array}$} & 0 tros accidentes, incluso los efectos tardíos & 14168 & 903 & 1032 & 1156 & 4983 & 3982 & 1926 & 186 \\
\hline & Accidentes debidos a factores naturales y del ambiente & 1066 & 70 & 132 & 137 & 256 & 264 & 191 & 16 \\
\hline & Ahogamiento y sumersión accidentales & 2818 & 44 & 446 & 449 & 1099 & 586 & 162 & 32 \\
\hline & Cuerpo extraño que penetra accidentalmente por un orificio natural & 3 & 0 & 0 & 0 & - & 1 & 2 & 0 \\
\hline & Accidente causado por maquinaria y por instrumentos cortantes & & & & & & & & \\
\hline & o punzantes & 286 & 1 & 5 & 12 & 154 & 93 & 17 & 4 \\
\hline E524 & Accidente causado por proyectil de arma de fuego & 916 & 0 & 12 & 65 & 541 & 251 & 27 & 20 \\
\hline E529 & 0 tros accidentes, incluso los efectos tardíos & 7737 & 788 & 437 & 493 & 2993 & 1445 & 1527 & 114 \\
\hline \multirow[t]{2}{*}{ E53 } & Drogas y medicamentos que causan efectos adversos & & & & & & & & \\
\hline & en su uso terapéutico & 124 & 18 & 11 & 9 & 32 & 35 & 18 & 1 \\
\hline
\end{tabular}

Fuente: Instituto N acional de Estadística, Geografía e Informática y Dirección General de Estadística e Informática de la Secretaría de Salud 


\section{Defunciones por accidentes Según CaUSAS de La lista básica y POR SeXo. MéXico, 1997}

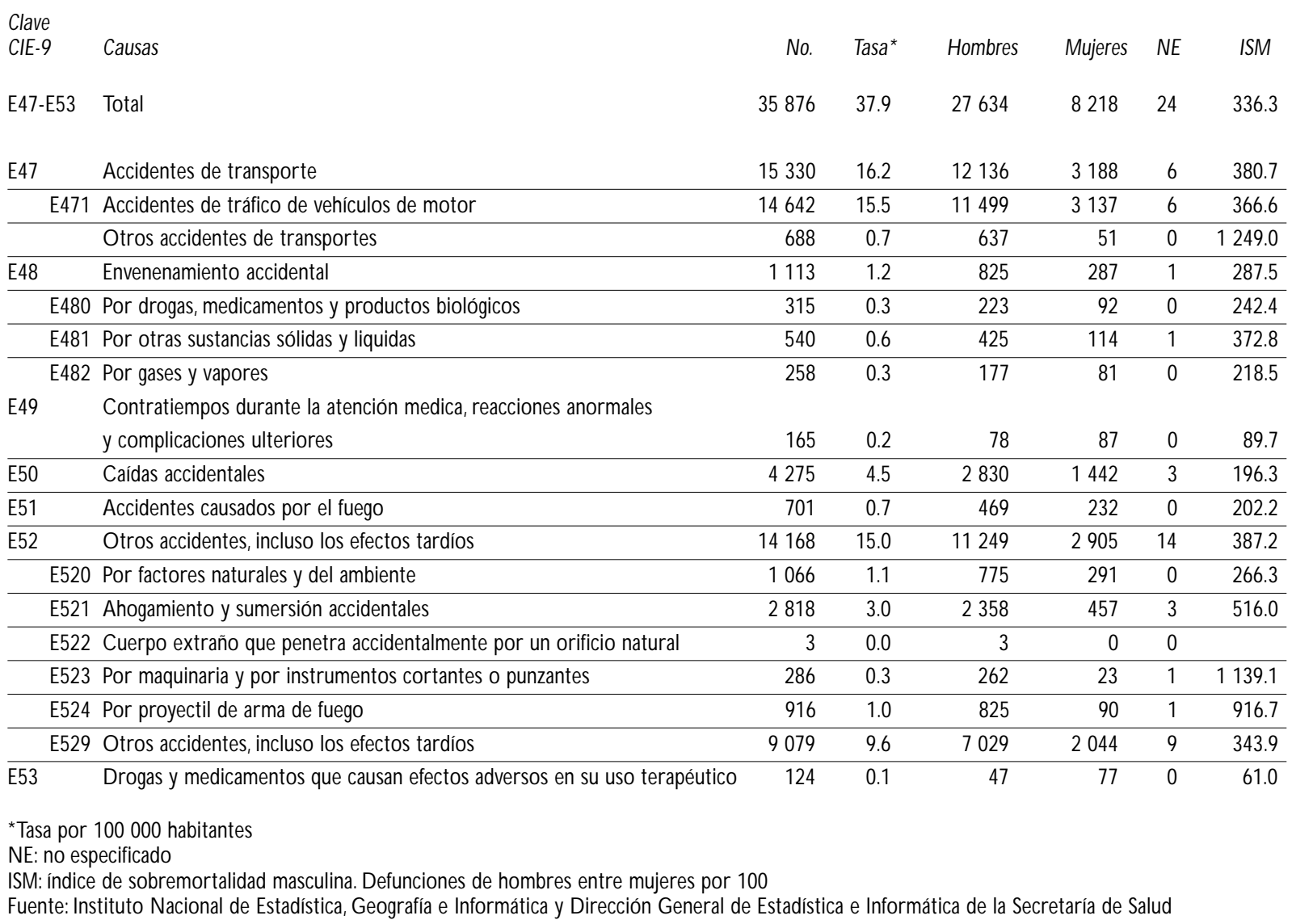




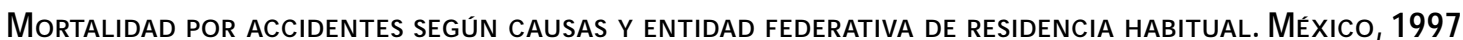

\begin{tabular}{|c|c|c|c|c|c|c|c|c|c|c|c|c|c|c|}
\hline \multirow[t]{2}{*}{ Entidad federativa } & \multicolumn{2}{|c|}{ Total } & \multicolumn{2}{|c|}{$\begin{array}{l}\text { De tráfico de ve- } \\
\text { hículo de motor }\end{array}$} & \multicolumn{2}{|c|}{$\begin{array}{l}\text { Envenenamiento } \\
\text { accidental }\end{array}$} & \multicolumn{2}{|c|}{$\begin{array}{c}\text { Caídas } \\
\text { accidentales }\end{array}$} & \multicolumn{2}{|c|}{$\begin{array}{l}\text { Ahogamiento } \\
\text { y sumersión }\end{array}$} & \multicolumn{2}{|c|}{$\begin{array}{l}\text { Por proyectil de } \\
\text { arma de fuego }\end{array}$} & \multicolumn{2}{|c|}{ Las demás } \\
\hline & $\begin{array}{l}\text { Defun- } \\
\text { ciones }\end{array}$ & Tasa* & $\begin{array}{l}\text { Defun- } \\
\text { ciones }\end{array}$ & Tasa* & $\begin{array}{l}\text { Defun- } \\
\text { ciones }\end{array}$ & Tasa* & $\begin{array}{l}\text { Defun- } \\
\text { ciones }\end{array}$ & Tasa* & $\begin{array}{l}\text { Defun- } \\
\text { ciones }\end{array}$ & Tasa* & $\begin{array}{l}\text { Defun- } \\
\text { ciones }\end{array}$ & Tasa* & $\begin{array}{l}\text { Defun- } \\
\text { ciones }\end{array}$ & Tasa* \\
\hline Estados Unidos Mexicanos & 35876 & 37.9 & 14642 & 15.5 & 1113 & 1.2 & 4275 & 4.5 & 2818 & 3.0 & 916 & 1.0 & 12112 & 12.8 \\
\hline Aguascalientes & 351 & 39.9 & 163 & 18.5 & 9 & 1.0 & 44 & 5.0 & 26 & 3.0 & 8 & 0.9 & 101 & 11.5 \\
\hline Baja California & 1257 & 55.5 & 273 & 12.0 & 174 & 7.7 & 31 & 1.4 & 80 & 3.5 & 110 & 4.9 & 589 & 26.0 \\
\hline Baja California Sur & 173 & 42.3 & 111 & 27.2 & 4 & 1.0 & 14 & 3.4 & 17 & 4.2 & 0 & 0.0 & 27 & 6.6 \\
\hline Campeche & 264 & 39.9 & 121 & 18.3 & 3 & 0.5 & 30 & 4.5 & 26 & 3.9 & 4 & 0.6 & 80 & 12.1 \\
\hline Coahuila & 835 & 37.1 & 328 & 14.6 & 29 & 1.3 & 89 & 4.0 & 53 & 2.4 & 26 & 1.2 & 310 & 13.8 \\
\hline Colima & 222 & 45.5 & 107 & 21.9 & 2 & 0.4 & 26 & 5.3 & 21 & 4.3 & 3 & 0.6 & 63 & 12.9 \\
\hline Chiapas & 1621 & 42.1 & 306 & 8.0 & 53 & 1.4 & 136 & 3.5 & 132 & 3.4 & 166 & 4.3 & 828 & 21.5 \\
\hline Chihuahua & 1587 & 54.2 & 622 & 21.2 & 177 & 6.0 & 122 & 4.2 & 78 & 2.7 & 83 & 2.8 & 505 & 17.2 \\
\hline Distrito Federal & 2550 & 31.9 & 1264 & 15.8 & 28 & 0.3 & 528 & 6.6 & 98 & 1.2 & 20 & 0.2 & 612 & 7.6 \\
\hline Durango & 638 & 44.1 & 194 & 13.4 & 12 & 0.8 & 74 & 5.1 & 41 & 2.8 & 54 & 3.7 & 263 & 18.2 \\
\hline Guanajuato & 1697 & 37.0 & 882 & 19.2 & 28 & 0.6 & 200 & 4.4 & 134 & 2.9 & 24 & 0.5 & 429 & 9.4 \\
\hline Guerrero & 1330 & 45.4 & 432 & 14.8 & 46 & 1.6 & 104 & 3.6 & 67 & 2.3 & 63 & 2.2 & 618 & 21.1 \\
\hline Hidalgo & 882 & 40.7 & 293 & 13.5 & 24 & 1.1 & 136 & 6.3 & 82 & 3.8 & 13 & 0.6 & 334 & 15.4 \\
\hline Jalisco & 2532 & 39.5 & 1272 & 19.9 & 36 & 0.6 & 383 & 6.0 & 221 & 3.4 & 17 & 0.3 & 603 & 9.4 \\
\hline México & 3738 & 29.0 & 1908 & 14.8 & 74 & 0.6 & 469 & 3.6 & 224 & 1.7 & 24 & 0.2 & 1039 & 8.1 \\
\hline Michoacán & 1643 & 42.1 & 645 & 16.5 & 34 & 0.9 & 224 & 5.7 & 127 & 3.3 & 46 & 1.2 & 567 & 14.5 \\
\hline Morelos & 543 & 38.0 & 189 & 13.2 & 25 & 1.7 & 63 & 4.4 & 34 & 2.4 & 4 & 0.3 & 228 & 15.9 \\
\hline $\mathrm{N}$ ayarit & 384 & 42.4 & 169 & 18.7 & 5 & 0.6 & 39 & 4.3 & 19 & 2.1 & 4 & 0.4 & 148 & 16.3 \\
\hline Nuevo León & 1187 & 33.1 & 337 & 9.4 & 29 & 0.8 & 82 & 2.3 & 50 & 1.4 & 38 & 1.1 & 651 & 18.1 \\
\hline 0 axaca & 1380 & 39.9 & 536 & 15.5 & 19 & 0.5 & 187 & 5.4 & 156 & 4.5 & 34 & 1.0 & 448 & 12.9 \\
\hline Puebla & 1727 & 34.6 & 484 & 9.7 & 64 & 1.3 & 194 & 3.9 & 130 & 2.6 & 22 & 0.4 & 833 & 16.7 \\
\hline Querétaro & 456 & 35.0 & 205 & 15.7 & 8 & 0.6 & 74 & 5.7 & 43 & 3.3 & 3 & 0.2 & 123 & 9.4 \\
\hline Q uintana Roo & 258 & 34.9 & 96 & 13.0 & 6 & 0.8 & 21 & 2.8 & 34 & 4.6 & 8 & 1.1 & 93 & 12.6 \\
\hline San Luis Potosí & 767 & 33.6 & 311 & 13.6 & 28 & 1.2 & 90 & 3.9 & 55 & 2.4 & 4 & 0.2 & 279 & 12.2 \\
\hline Sinaloa & 1056 & 43.2 & 519 & 21.3 & 26 & 1.1 & 93 & 3.8 & 134 & 5.5 & 11 & 0.5 & 273 & 11.2 \\
\hline Sonora & 1015 & 47.8 & 475 & 22.4 & 54 & 2.5 & 91 & 4.3 & 57 & 2.7 & 41 & 1.9 & 297 & 14.0 \\
\hline Tabasco & 793 & 44.9 & 322 & 18.2 & 8 & 0.5 & 68 & 3.9 & 160 & 9.1 & 7 & 0.4 & 228 & 12.9 \\
\hline Tamaulipas & 950 & 36.5 & 480 & 18.4 & 15 & 0.6 & 112 & 4.3 & 90 & 3.5 & 14 & 0.5 & 239 & 9.2 \\
\hline Tlaxcala & 346 & 37.2 & 139 & 15.0 & 17 & 1.8 & 32 & 3.4 & 21 & 2.3 & 3 & 0.3 & 134 & 14.4 \\
\hline Veracruz & 1920 & 26.8 & 683 & 9.5 & 18 & 0.3 & 361 & 5.0 & 256 & 3.6 & 11 & 0.2 & 591 & 8.2 \\
\hline Yucatán & 488 & 31.3 & 202 & 12.9 & 5 & 0.3 & 62 & 4.0 & 40 & 2.6 & 6 & 0.4 & 173 & 11.1 \\
\hline Zacatecas & 652 & 47.8 & 283 & 20.7 & 21 & 1.5 & 79 & 5.8 & 35 & 2.6 & 14 & 1.0 & 220 & 16.1 \\
\hline Extranjero & 634 & & 291 & & 32 & & 17 & & 77 & & 31 & & 18 & \\
\hline
\end{tabular}

*Tasa por 100000 habitantes

Fuente: Instituto N acional de Estadística, Geografía e Informática y Dirección de General de Estadística e Informática de la Secretaría de Salud 


\section{Cuadro VI}

\section{MORTALIDAD POR ACCIDENTES, PESO RELATIVO Y LUGAR QUE OCUPAN DENTRO DE LAS PRINCIPALES CAUSAS DE Defunción de CADA EnTIDAd. MÉxico, 1997}

\begin{tabular}{|c|c|c|c|c|}
\hline Entidad federativa & Total & Tasa* & Peso relativo $0^{\ddagger}$ & Número de orden \\
\hline Estados U nidos Mexicanos & 35876 & 37.9 & 8.1 & 4 \\
\hline Aguascalientes & 351 & 39.9 & 9.3 & 3 \\
\hline Baja California & 1257 & 55.5 & 11.9 & 2 \\
\hline Baja California Sur & 173 & 42.3 & 10.6 & 3 \\
\hline Campeche & 264 & 39.9 & 10.3 & 3 \\
\hline Coahuila & 835 & 37.1 & 8.1 & 4 \\
\hline Colima & 222 & 45.5 & 9.2 & 3 \\
\hline Chiapas & 1621 & 42.1 & 10.4 & 1 \\
\hline Chihuahua & 1587 & 54.2 & 10.2 & 3 \\
\hline Distrito Federal & 2550 & 31.9 & 5.4 & 5 \\
\hline Durango & 638 & 44.1 & 10.4 & 3 \\
\hline Guanajuato & 1697 & 37.0 & 7.9 & 4 \\
\hline Guerrero & 1330 & 45.4 & 11.9 & 2 \\
\hline Hidalgo & 882 & 40.7 & 9.0 & 3 \\
\hline Jalisco & 2532 & 39.5 & 8.2 & 4 \\
\hline México & 3738 & 29.0 & 7.3 & 5 \\
\hline Michoacán & 1643 & 42.1 & 8.8 & 3 \\
\hline Morelos & 543 & 38.0 & 8.1 & 4 \\
\hline $\mathrm{N}$ ayarit & 384 & 42.4 & 9.4 & 3 \\
\hline Nuevo León & 1187 & 33.1 & 7.6 & 4 \\
\hline 0 axaca & 1380 & 39.9 & 7.5 & 3 \\
\hline Puebla & 1727 & 34.6 & 6.5 & 7 \\
\hline Q uerétaro & 456 & 35.0 & 8.0 & 3 \\
\hline Q uintana Roo & 258 & 34.9 & 12.6 & 1 \\
\hline San Luis Potosí & 767 & 33.6 & 7.5 & 3 \\
\hline Sinaloa & 1056 & 43.2 & 10.6 & 3 \\
\hline Sonora & 1015 & 47.8 & 9.4 & 3 \\
\hline Tabasco & 793 & 44.9 & 11.0 & 3 \\
\hline Tamaulipas & 950 & 36.5 & 8.3 & 4 \\
\hline Tlaxcala & 346 & 37.2 & 7.4 & 4 \\
\hline Veracruz & 1920 & 26.8 & 6.1 & 6 \\
\hline Yucatán & 488 & 31.3 & 6.1 & 6 \\
\hline Zacatecas & 652 & 47.8 & 10.0 & 3 \\
\hline
\end{tabular}

Extranjeros

634

*Tasa por 100000 habitantes

¥Porcentaje respecto al total de defunciones de cada entidad

Fuente: Instituto N acional de Estadística, Geografía e Informática y Dirección General de Estadística e Informática de la Secretaría de Salud 
Cuadro VII

Defunciones POR ACCIDENTES Y SITIO dONDE OCURRIó la DEFUNCIÓN, SEGÚN ENTIDAD FEDERATIVA De Residencia habitual. MÉXICO, 1997

\begin{tabular}{|c|c|c|c|c|c|c|c|c|c|}
\hline \multirow[t]{2}{*}{ Entidad federatuva } & \multirow[b]{2}{*}{ Total } & \multicolumn{2}{|c|}{ Unidad médica } & \multicolumn{2}{|c|}{ Hogar } & \multicolumn{2}{|c|}{ Otro } & \multicolumn{2}{|c|}{ No especificado } \\
\hline & & No. & $\% *$ & No. & $\% *$ & No. & $\% *$ & No. & $\% *$ \\
\hline Estados Unidos Mexicanos & 35876 & 10640 & 29.7 & 4841 & 13.5 & 16629 & 46.4 & 3766 & 10.5 \\
\hline Aguascalientes & 351 & 99 & 28.2 & 60 & 17.1 & 147 & 41.9 & 45 & 12.8 \\
\hline Baja California & 1257 & 478 & 38.0 & 177 & 14.1 & 597 & 47.5 & 5 & 0.4 \\
\hline Baja C alifornia Sur & 173 & 50 & 28.9 & 12 & 6.9 & 110 & 63.6 & 1 & 0.6 \\
\hline Campeche & 264 & 73 & 27.7 & 34 & 12.9 & 144 & 54.5 & 13 & 4.9 \\
\hline Coahuila & 835 & 238 & 28.5 & 88 & 10.5 & 324 & 38.8 & 185 & 22.2 \\
\hline Colima & 222 & 86 & 38.7 & 20 & 9.0 & 102 & 45.9 & 14 & 6.3 \\
\hline Chiapas & 1621 & 309 & 19.1 & 326 & 20.1 & 859 & 53.0 & 127 & 7.8 \\
\hline Chihuahua & 1587 & 377 & 23.8 & 189 & 11.9 & 517 & 32.6 & 504 & 31.8 \\
\hline Distrito Federal & 2550 & 1245 & 48.8 & 222 & 8.7 & 1039 & 40.7 & 44 & 1.7 \\
\hline Durango & 638 & 144 & 22.6 & 66 & 10.3 & 241 & 37.8 & 187 & 29.3 \\
\hline Guanajuato & 1697 & 458 & 27.0 & 240 & 14.1 & 871 & 51.3 & 128 & 7.5 \\
\hline Guerrero & 1330 & 309 & 23.2 & 324 & 24.4 & 485 & 36.5 & 212 & 15.9 \\
\hline Hidalgo & 882 & 213 & 24.1 & 169 & 19.2 & 434 & 49.2 & 66 & 7.5 \\
\hline Jalisco & 2532 & 827 & 32.7 & 353 & 13.9 & 1197 & 47.3 & 155 & 6.1 \\
\hline México & 3738 & 1229 & 32.9 & 416 & 11.1 & 1788 & 47.8 & 305 & 8.2 \\
\hline Michoacán & 1643 & 414 & 25.2 & 262 & 15.9 & 733 & 44.6 & 234 & 14.2 \\
\hline Morelos & 543 & 232 & 42.7 & 60 & 11.0 & 181 & 33.3 & 70 & 12.9 \\
\hline $\mathrm{N}$ ayarit & 384 & 94 & 24.5 & 44 & 11.5 & 182 & 47.4 & 64 & 16.7 \\
\hline N uevo León & 1187 & 305 & 25.7 & 94 & 7.9 & 289 & 24.3 & 499 & 42.0 \\
\hline 0 axaca & 1380 & 290 & 21.0 & 278 & 20.1 & 630 & 45.7 & 182 & 13.2 \\
\hline Puebla & 1727 & 528 & 30.6 & 287 & 16.6 & 864 & 50.0 & 48 & 2.8 \\
\hline Q uerétaro & 456 & 132 & 28.9 & 60 & 13.2 & 255 & 55.9 & 9 & 2.0 \\
\hline Q uintana Roo & 258 & 67 & 26.0 & 30 & 11.6 & 99 & 38.4 & 62 & 24.0 \\
\hline San Luis Potosí & 767 & 199 & 25.9 & 120 & 15.6 & 377 & 49.2 & 71 & 9.3 \\
\hline Sinaloa & 1056 & 307 & 29.1 & 125 & 11.8 & 615 & 58.2 & 9 & 0.9 \\
\hline Sonora & 1015 & 326 & 32.1 & 120 & 11.8 & 567 & 55.9 & 2 & 0.2 \\
\hline Tabasco & 793 & 180 & 22.7 & 84 & 10.6 & 468 & 59.0 & 61 & 7.7 \\
\hline Tamaulipas & 950 & 273 & 28.7 & 76 & 8.0 & 440 & 46.3 & 161 & 16.9 \\
\hline Tlaxcala & 346 & 107 & 30.9 & 63 & 18.2 & 169 & 48.8 & 7 & 2.0 \\
\hline Veracruz & 1920 & 587 & 30.6 & 218 & 11.4 & 1029 & 53.6 & 86 & 4.5 \\
\hline Yucatán & 488 & 169 & 34.6 & 73 & 15.0 & 229 & 46.9 & 17 & 3.5 \\
\hline Zacatecas & 652 & 155 & 23.8 & 134 & 20.6 & 303 & 46.5 & 60 & 9.2 \\
\hline Extranjeros & 634 & 140 & 22.1 & 17 & 2.7 & 344 & 54.3 & 133 & 21.0 \\
\hline
\end{tabular}




\section{Cuadro VIII \\ DEFUnCIONES POR ACCIDENTES Y LUGAR DONDE OCURRIÓ LA LESIÓN, SEGÚN ENTIDAD FEDERATIVA De Residencia habitual. MÉXICO, 1997}

\begin{tabular}{|c|c|c|c|c|c|c|c|c|c|c|c|}
\hline \multirow{2}{*}{ Entidad federativa } & \multirow[b]{2}{*}{ Total } & \multicolumn{2}{|c|}{ Hogar } & \multicolumn{2}{|c|}{ Trabajo } & \multicolumn{2}{|c|}{ Vía pública } & \multicolumn{2}{|c|}{ Otros } & \multicolumn{2}{|c|}{ No especificado } \\
\hline & & No. & \%* & No. & \%* & No. & \%* & No. & \%* & No. & \%* \\
\hline Estados Unidos Mexicanos & 35876 & 2848 & 7.9 & 2628 & 7.3 & 15759 & 43.9 & 4491 & 12.5 & 10150 & 28.3 \\
\hline A guascalientes & 351 & 9 & 2.6 & 14 & 4.0 & 123 & 35.0 & 37 & 10.5 & 168 & 47.9 \\
\hline Baja California & 1257 & 65 & 5.2 & 23 & 1.8 & 301 & 23.9 & 72 & 5.7 & 796 & 63.3 \\
\hline Baja California Sur & 173 & 5 & 2.9 & 14 & 8.1 & 106 & 61.3 & 25 & 14.5 & 23 & 13.3 \\
\hline Campeche & 264 & 22 & 8.3 & 20 & 7.6 & 133 & 50.4 & 35 & 13.3 & 54 & 20.5 \\
\hline Coahuila & 835 & 51 & 6.1 & 57 & 6.8 & 437 & 52.3 & 46 & 5.5 & 244 & 29.2 \\
\hline Colima & 222 & 16 & 7.2 & 13 & 5.9 & 106 & 47.7 & 44 & 19.8 & 43 & 19.4 \\
\hline Chiapas & 1621 & 40 & 2.5 & 29 & 1.8 & 202 & 12.5 & 70 & 4.3 & 1280 & 79.0 \\
\hline Chihuahua & 1587 & 134 & 8.4 & 102 & 6.4 & 628 & 39.6 & 143 & 9.0 & 580 & 36.5 \\
\hline Distrito Federal & 2550 & 338 & 13.3 & 289 & 11.3 & 1412 & 55.4 & 233 & 9.1 & 278 & 10.9 \\
\hline Durango & 638 & 20 & 3.1 & 50 & 7.8 & 254 & 39.8 & 61 & 9.6 & 253 & 39.7 \\
\hline Guanajuato & 1697 & 137 & 8.1 & 93 & 5.5 & 862 & 50.8 & 265 & 15.6 & 340 & 20.0 \\
\hline Guerrero & 1330 & 156 & 11.7 & 139 & 10.5 & 428 & 32.2 & 190 & 14.3 & 417 & 31.4 \\
\hline Hidalgo & 882 & 44 & 5.0 & 80 & 9.1 & 305 & 34.6 & 111 & 12.6 & 342 & 38.8 \\
\hline Jalisco & 2532 & 342 & 13.5 & 191 & 7.5 & 1150 & 45.4 & 448 & 17.7 & 401 & 15.8 \\
\hline México & 3738 & 405 & 10.8 & 315 & 8.4 & 2090 & 55.9 & 414 & 11.1 & 514 & 13.8 \\
\hline Michoacán & 1643 & 162 & 9.9 & 110 & 6.7 & 762 & 46.4 & 252 & 15.3 & 357 & 21.7 \\
\hline Morelos & 543 & 57 & 10.5 & 50 & 9.2 & 253 & 46.6 & 73 & 13.4 & 110 & 20.3 \\
\hline N ayarit & 384 & 29 & 7.6 & 26 & 6.8 & 173 & 45.1 & 61 & 15.9 & 95 & 24.7 \\
\hline Nuevo León & 1187 & 33 & 2.8 & 62 & 5.2 & 309 & 26.0 & 39 & 3.3 & 744 & 62.7 \\
\hline 0 axaca & 1380 & 133 & 9.6 & 105 & 7.6 & 488 & 35.4 & 300 & 21.7 & 354 & 25.7 \\
\hline Puebla & 1727 & 90 & 5.2 & 166 & 9.6 & 846 & 49.0 & 194 & 11.2 & 431 & 25.0 \\
\hline Q uerétaro & 456 & 31 & 6.8 & 42 & 9.2 & 233 & 51.1 & 81 & 17.8 & 69 & 15.1 \\
\hline Q uintana Roo & 258 & 15 & 5.8 & 18 & 7.0 & 79 & 30.6 & 32 & 12.4 & 114 & 44.2 \\
\hline San Luis Potosí & 767 & 32 & 4.2 & 88 & 11.5 & 384 & 50.1 & 99 & 12.9 & 164 & 21.4 \\
\hline Sinaloa & 1056 & 47 & 4.5 & 58 & 5.5 & 502 & 47.5 & 155 & 14.7 & 294 & 27.8 \\
\hline Sonora & 1015 & 82 & 8.1 & 46 & 4.5 & 490 & 48.3 & 117 & 11.5 & 280 & 27.6 \\
\hline Tabasco & 793 & 60 & 7.6 & 42 & 5.3 & 411 & 51.8 & 124 & 15.6 & 156 & 19.7 \\
\hline Tamaulipas & 950 & 52 & 5.5 & 76 & 8.0 & 507 & 53.4 & 102 & 10.7 & 213 & 22.4 \\
\hline Tlaxcala & 346 & 29 & 8.4 & 28 & 8.1 & 191 & 55.2 & 31 & 9.0 & 67 & 19.4 \\
\hline Veracruz & 1920 & 134 & 7.0 & 167 & 8.7 & 855 & 44.5 & 397 & 20.7 & 367 & 19.1 \\
\hline Yucatán & 488 & 29 & 5.9 & 33 & 6.8 & 235 & 48.2 & 61 & 12.5 & 130 & 26.6 \\
\hline Zacatecas & 652 & 41 & 6.3 & 72 & 11.0 & 298 & 45.7 & 72 & 11.0 & 169 & 25.9 \\
\hline Extranjeros & 634 & 8 & 1.3 & 10 & 1.6 & 206 & 32.5 & 107 & 16.9 & 303 & 47.8 \\
\hline
\end{tabular}




\section{Cuadro IX \\ DeFunCiONeS POR ACCIDENTES Y MES EN EL QUE OCURRIERON, SEGÚN ENTIDAD FEDERATIVA De Residencia habitual. México 1997}

\begin{tabular}{|c|c|c|c|c|c|c|c|c|c|c|c|c|c|c|}
\hline Entidad federativa & Total & Ene. & Feb. & Mar. & Abr. & May. & Jun. & Jul. & Ago. & Sep. & Oct. & Nov. & Dic. & $\mathrm{NE}$ \\
\hline Estados Unidos Mexicanos & 35876 & 2923 & 2621 & 3120 & 2741 & 3045 & 2898 & 3100 & 3130 & 2804 & 3108 & 2856 & 3377 & 153 \\
\hline Aguascalientes & 351 & 40 & 19 & 29 & 27 & 32 & 29 & 30 & 36 & 28 & 26 & 22 & 33 & 0 \\
\hline Baja California & 1257 & 95 & 97 & 136 & 99 & 113 & 94 & 99 & 121 & 106 & 94 & 87 & 113 & 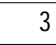 \\
\hline Baja California Sur & 173 & 15 & 8 & 11 & 6 & 14 & 15 & 23 & 22 & 17 & 14 & 16 & 12 & 0 \\
\hline Campeche & 264 & 13 & 21 & 31 & 19 & 17 & 25 & 26 & 20 & 18 & 19 & 25 & 29 & 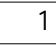 \\
\hline Coahuila & 835 & 81 & 55 & 60 & 59 & 64 & 84 & 88 & 69 & 55 & 73 & 59 & 85 & 3 \\
\hline Colima & 222 & 17 & 23 & 20 & 22 & 28 & 19 & 16 & 25 & 11 & 9 & 19 & 13 & 0 \\
\hline Chiapas & 1621 & 109 & 109 & 152 & 161 & 144 & 150 & 158 & 128 & 119 & 86 & 152 & 124 & 29 \\
\hline Chihuahua & 1587 & 119 & 114 & 131 & 110 & 129 & 137 & 134 & 145 & 110 & 136 & 133 & 173 & 16 \\
\hline Distrito Federal & 2550 & 191 & 174 & 265 & 209 & 186 & 170 & 218 & 223 & 217 & 220 & 191 & 284 & 2 \\
\hline Durango & 638 & 60 & 52 & 46 & 58 & 57 & 52 & 59 & 59 & 45 & 38 & 53 & 55 & 4 \\
\hline Guanajuato & 1697 & 149 & 150 & 141 & 122 & 143 & 132 & 140 & 150 & 124 & 142 & 147 & 154 & 3 \\
\hline Guerrero & 1330 & 103 & 95 & 94 & 83 & 141 & 85 & 105 & 102 & 91 & 227 & 92 & 109 & 3 \\
\hline Hidalgo & 882 & 73 & 57 & 67 & 63 & 56 & 71 & 74 & 80 & 85 & 88 & 77 & 79 & 12 \\
\hline Jalisco & 2532 & 213 & 211 & 220 & 204 & 208 & 202 & 197 & 218 & 193 & 223 & 214 & 222 & 7 \\
\hline México & 3738 & 297 & 265 & 321 & 287 & 276 & 315 & 323 & 330 & 318 & 335 & 264 & 397 & 10 \\
\hline Michoacán & 1643 & 176 & 124 & 128 & 119 & 174 & 139 & 128 & 136 & 138 & 117 & 122 & 128 & 14 \\
\hline Morelos & 543 & 50 & 40 & 39 & 52 & 51 & 43 & 41 & 56 & 40 & 52 & 37 & 42 & 0 \\
\hline $\mathrm{N}$ ayarit & 384 & 35 & 26 & 21 & 24 & 43 & 32 & 37 & 33 & 23 & 36 & 35 & 37 & 2 \\
\hline Nuevo León & 1187 & 87 & 88 & 91 & 79 & 83 & 86 & 122 & 108 & 123 & 120 & 103 & 96 & 1 \\
\hline 0 axaca & 1380 & 106 & 94 & 109 & 128 & 108 & 112 & 111 & 87 & 94 & 182 & 107 & 137 & 5 \\
\hline Puebla & 1727 & 147 & 133 & 138 & 130 & 152 & 105 & 160 & 146 & 129 & 143 & 155 & 178 & 11 \\
\hline Q uerétaro & 456 & 45 & 26 & 43 & 31 & 45 & 41 & 44 & 33 & 40 & 33 & 33 & 42 & 0 \\
\hline Q uintana Roo & 258 & 23 & 19 & 16 & 18 & 36 & 20 & 14 & 23 & 18 & 20 & 31 & 20 & 0 \\
\hline San Luis Potosí & 767 & 61 & 64 & 55 & 72 & 65 & 63 & 70 & 80 & 46 & 61 & 62 & 65 & 3 \\
\hline Sinaloa & 1056 & 86 & 87 & 88 & 76 & 90 & 82 & 92 & 100 & 98 & 82 & 83 & 90 & 2 \\
\hline Sonora & 1015 & 74 & 76 & 86 & 74 & 96 & 78 & 75 & 110 & 87 & 77 & 98 & 81 & 3 \\
\hline Tabasco & 793 & 53 & 52 & 81 & 66 & 69 & 69 & 83 & 68 & 49 & 65 & 57 & 80 & 1 \\
\hline Tamaulipas & 950 & 85 & 61 & 88 & 60 & 81 & 87 & 89 & 79 & 75 & 80 & 52 & 110 & 3 \\
\hline Tlaxcala & 346 & 31 & 21 & 28 & 17 & 30 & 26 & 22 & 31 & 31 & 28 & 39 & 42 & 0 \\
\hline Veracruz & 1920 & 147 & 143 & 214 & 145 & 167 & 180 & 158 & 154 & 142 & 156 & 154 & 152 & 8 \\
\hline Yucatán & 488 & 32 & 36 & 54 & 31 & 39 & 45 & 37 & 48 & 31 & 37 & 47 & 51 & 0 \\
\hline Zacatecas & 652 & 57 & 39 & 54 & 47 & 59 & 46 & 72 & 54 & 59 & 51 & 53 & 59 & 2 \\
\hline Extranjeros & 634 & 53 & 42 & 63 & 43 & 49 & 64 & 55 & 56 & 44 & 38 & 37 & 85 & 5 \\
\hline
\end{tabular}

NE: no especificado

Fuente: Instituto N acional de Estadística, Geografía e Informática y D irección General de Estadística e Informática de la Secretaría de Salud 


\section{Cuadro $X$ \\ Defunciones por accidentes y Condición De derechohabiencia, Según entidad federativa De Residencia habitual. MÉXICO, 1997}

\begin{tabular}{|c|c|c|c|c|c|c|c|}
\hline \multirow[t]{2}{*}{ Entidad federativa } & \multirow[b]{2}{*}{ Total } & \multicolumn{2}{|c|}{ Derechohabiente } & \multicolumn{2}{|c|}{ No derechohabiente } & \multicolumn{2}{|c|}{ NE } \\
\hline & & No. & $\% *$ & No. & $\% *$ & No. & $\% *$ \\
\hline Estados Unidos Mexicanos & 35876 & 11198 & 31.2 & 17215 & 48.0 & 7463 & 20.8 \\
\hline A guascalientes & 351 & 176 & 50.1 & 144 & 41.0 & 31 & 8.8 \\
\hline Baja California & 1257 & 407 & 32.4 & 610 & 48.5 & 240 & 19.1 \\
\hline Baja California Sur & 173 & 94 & 54.3 & 73 & 42.2 & 6 & 3.5 \\
\hline Campeche & 264 & 89 & 33.7 & 136 & 51.5 & 39 & 14.8 \\
\hline Coahuila & 835 & 448 & 53.7 & 221 & 26.5 & 166 & 19.9 \\
\hline Colima & 222 & 75 & 33.8 & 120 & 54.1 & 27 & 12.2 \\
\hline Chiapas & 1621 & 80 & 4.9 & 305 & 18.8 & 1236 & 76.2 \\
\hline Chihuahua & 1587 & 359 & 22.6 & 427 & 26.9 & 801 & 50.5 \\
\hline Distrito Federal & 2550 & 1227 & 48.1 & 1177 & 46.2 & 146 & 5.7 \\
\hline Durango & 638 & 243 & 38.1 & 167 & 26.2 & 228 & 35.7 \\
\hline Guanajuato & 1697 & 458 & 27.0 & 1024 & 60.3 & 215 & 12.7 \\
\hline Guerrero & 1330 & 274 & 20.6 & 687 & 51.7 & 369 & 27.7 \\
\hline Hidalgo & 882 & 187 & 21.2 & 448 & 50.8 & 247 & 28.0 \\
\hline Jalisco & 2532 & 880 & 34.8 & 1326 & 52.4 & 326 & 12.9 \\
\hline México & 3738 & 1339 & 35.8 & 1978 & 52.9 & 421 & 11.3 \\
\hline Michoacán & 1643 & 320 & 19.5 & 1037 & 63.1 & 286 & 17.4 \\
\hline Morelos & 543 & 197 & 36.3 & 284 & 52.3 & 62 & 11.4 \\
\hline $\mathrm{N}$ ayarit & 384 & 121 & 31.5 & 172 & 44.8 & 91 & 23.7 \\
\hline Nuevo León & 1187 & 455 & 38.3 & 228 & 19.2 & 504 & 42.5 \\
\hline 0 axaca & 1380 & 260 & 18.8 & 878 & 63.6 & 242 & 17.5 \\
\hline Puebla & 1727 & 415 & 24.0 & 1011 & 58.5 & 301 & 17.4 \\
\hline Q uerétaro & 456 & 156 & 34.2 & 277 & 60.7 & 23 & 5.0 \\
\hline Q uintana Roo & 258 & 85 & 32.9 & 97 & 37.6 & 76 & 29.5 \\
\hline San Luis Potosí & 767 & 211 & 27.5 & 460 & 60.0 & 96 & 12.5 \\
\hline Sinaloa & 1056 & 492 & 46.6 & 457 & 43.3 & 107 & 10.1 \\
\hline Sonora & 1015 & 426 & 42.0 & 456 & 44.9 & 133 & 13.1 \\
\hline Tabasco & 793 & 175 & 22.1 & 503 & 63.4 & 115 & 14.5 \\
\hline Tamaulipas & 950 & 368 & 38.7 & 408 & 42.9 & 174 & 18.3 \\
\hline Tlaxcala & 346 & 112 & 32.4 & 209 & 60.4 & 25 & 7.2 \\
\hline Veracruz & 1920 & 596 & 31.0 & 1115 & 58.1 & 209 & 10.9 \\
\hline Yucatán & 488 & 215 & 44.1 & 224 & 45.9 & 49 & 10.0 \\
\hline Zacatecas & 652 & 165 & 25.3 & 384 & 58.9 & 103 & 15.8 \\
\hline Extranjeros & 634 & 93 & 14.7 & 172 & 27.1 & 369 & 58.2 \\
\hline
\end{tabular}

* Porcentaje respecto al total de defunciones de cada entidad $N E$ : no especificado

Fuente: Instituto N acional de Estadística, Geografía e Informática y Dirección General de Estadística e Informática de la Secretaría de Salud 